\title{
Modelagem Computacional para Produção e Pesquisa em Avicultura
}

Computer Modeling for Poultry Production and Research

Código / Code

$00 ? ?$

Autor(es) / Author(s)

Rondón $\mathrm{EOO}^{1}$

Murakami $\mathrm{AE}^{2}$

Sakaguti $\mathrm{ES}^{2}$

1-Doutorando no Department of Poultry Science, University of Arkansas, USA

2- Profo do Depto. de Zootecnia, Universidade Estadual de Maringá

\section{Correspondência / Mail Address}

??

Universidade Estadual de Maringá

Av. Colombo, 5790

87020-900 - Maringá - Paraná - Brasil

E-mail: ??

(aemurakami@uem.br ou essakaguti@uem.br)

\section{RESUMO}

A modelagem computacional foi apresentada como uma ferramenta na tomada de decisões para solucionar problemas complexos e cotidianos na produção e pesquisa em avicultura. Aspectos teóricos relacionados à construção e implementação dos diferentes tipos de modelos matemáticos utilizados em avicultura foram descritos de maneira elementar, tentando deixar 0 artigo acessível às pessoas que não necessariamente possuem um forte conhecimento de estatística experimental. Uma ênfase especial foi dada à discussão das vantagens da modelagem para resolver o problema complexo de determinar exigências nutricionais das aves em diferentes condições. Uma revisão cronológica das publicações relacionadas aos pacotes computacionais desenvolvidos para auxiliar na formulação de estratégias nutricionais em aves mostrou que eles estão baseados na modelagem do crescimento associada aos modelos de estimação de exigências e de custo mínimo de rações. A evolução e sofisticação desses programas têm acompanhado os rápidos avanços computacionais. Contudo, a eficiência de uma simulação depende dos conhecimentos sobre situações reais. Esses conhecimentos são gerados pelas pesquisas tradicionais e muitas vezes é de fundamental importância a conexão de informações provenientes de diferentes disciplinas, direta ou indiretamente, relacionadas à avicultura.

\section{ABSTRACT}

Computational modeling was presented as a tool to make decisions for solving the daily complex problems in poultry production and research. Theoretical aspects related to construction and implementation of the different types of models used in poultry production and research were elementarily described. We tried to make this paper accessible to people that do not necessarily have a strong background in experimental statistic. Special emphasis was given to discuss the advantages of modeling to solve the complex problem of nutrient requirement estimation for poultry under different conditions. A chronological review of publications related to software which was developed to help the establishment of poultry nutritional strategies show ed that they are based on growth modeling associated with models of nutritional requirement and minimum cost diets. The evolution and sophistication of those programs are coming with the advance of information technology. However, the efficiency of a simulation depends on the real situation knowledge that is provided by the traditional research. In many cases, it is fundamentally important to connect information of different disciplines that are direct or indirectly related with poultry production. 


\section{INTRODUÇÃO}

$\mathrm{Na}$ avicultura, os sistemas de integração são caracterizados pela subdivisão dos setores de produção como matrizes, incubatório, recria ou engorda. Essa subdivisão favorece a identificação e o controle de pontos críticos do processo de produção. Entretanto, em geral, devido à complexidade das interações entre os muitos fatores que afetam a cadeia produtiva, é praticamente impossível para a mente humana predizer, com alguma certeza, as conseqüências de uma implantação ou mudança de alguma estratégia de manejo, nutricional, de seleção, entre outras, para longo prazo e/ou para o sistema total de produção.

Uma alternativa para ajudar na tomada de decisões e definição de produtos melhores e mais econômicos, tanto na indústria como na pesquisa, é - uso da modelagem computacional. Essa metodologia procura transformar conceitos e conhecimentos pertinentes em equações matemáticas e implementá-las por meio de processos lógicos, simulando situações reais em computador. Dessa forma, as informações coletadas por indústrias e instituições de pesquisa podem ser aplicadas para avaliar sistemas avícolas comerciais (Spedding, 1988) e para definir prioridades de pesquisa (Black, 1995), com grande economia.

A partir de modelos biológicos razoáveis para um sistema em particular, pode-se ainda proceder simulações que permitam avaliações simultâneas de problemas estudados em vários projetos de pesquisa, as quais poderiam ser economicamente praticamente inviáveis em uma experimentação física.

Nos últimos vinte anos, em decorrência da evolução dos programas computacionais, das técnicas de modelagem e da capacidade de processamento dos computadores, ocorreu um grande aumento no número de pesquisas relacionadas ao desenvolvimento de modelos que tentam predizer as respostas das aves em diferentes tipos de situações. Conseqüentemente, vários programas que utilizam a modelagem computacional para tentar predizer o desempenho de sistemas biológicos e/ou de produção estão sendo lançados no mercado.

Várias opções podem ser empregadas para se representar um único fenômeno biológico. Por exemplo, o crescimento pode ser minuciosamente descrito pela hipertrofia e hiperplasia de células de tecidos específicos ou ser caracterizado pelo ganho de peso médio de uma linhagem. Assim, nota-se que diferentes programas podem ter diferentes formas de abordar o mesmo objetivo e os resultados dependem do tipo e da forma de implementação dos modelos.

o conhecimento dos processos envolvidos nos programas de simulação é fundamental para se identificar qual é o melhor programa para alcançar os objetivos almejados. Dessa forma, o presente artigo procurou mostrar, dentro do enfoque da avicultura, uma elementar e breve descrição teórica sobre modelagem e a aplicabilidade desta metodologia. Essa revisão deu maior ênfase à nutrição, embora seja notório o grande valor da modelagem no melhoramento genético, nos sistemas de manejo e na economia.

\section{Definição e classificação dos modelos}

Na pesquisa avícola, um "modelo" pode ser a descrição matemática, ou seja, por meio de equações, de fenômenos biológicos como crescimento, produção de ovos, incubação, digestão e absorção de nutrientes etc. Assim, variáveis quantitativas são tomadas para representar fatores que influenciam o fenômeno.

O modelo matemático mais simples que é empregado na pesquisa avícola é o modelo de um delineamento inteiramente ao acaso, dado por:

$$
y_{i j}=m+t_{j}+e_{i j}
$$

o qual assume que cada valor observado $\left(\mathrm{y}_{\mathrm{ij}}\right)$ é determinado por uma quantidade que é constante a todas unidades experimentais $(\mathrm{m})$ mais a quantidade que é comum às unidades que receberam o mesmo tratamento $\left(t_{i}\right)$ e ainda uma quantidade específica de cada observação $\left(e_{i j}\right)$.

Nota-se que toda a observação, de qualquer experimento, pode ser representada pelo modelo (1). Entretanto, a qualidade e a confiabilidade das informações extraídas com o auxílio do modelo vão depender de quanto o modelo se ajusta aos dados. Assim, em algumas ocasiões, é mais apropriada a utilização de modelos que descrevem um maior número de fatores, como tratamento, sexo e linhagem $\left(t_{i}, s_{j}\right.$ e $\left.I_{k}\right)$ e interações entre esses fatores $\left(t_{i j}, t_{i k}\right.$ e $\left.s l_{j k}\right)$ :

$$
y_{i j k}=m+t_{i}+s_{j}+I_{k}+t_{i j}+t_{i k}+s_{j k}+e_{i j k}
$$

Os modelos (1) e (2) podem ser classificados como modelos lineares. Um modelo é considerado linear quando todos os parâmetros estão linearmente dispostos, mesmo quando existirem termos elevados ao 
quadrado, ao cubo etc. Assim, outro exemplo de modelo linear é o modelo de regressão polinomial comumente empregado na determinação de exigências nutricionais:

$$
Y_{i j}=b_{0}+b_{1} X_{i}+b_{2} X_{i}^{2}+e_{i j}
$$

em que $y_{i j}$ pode ser o peso do animal j que recebeu 0 nível de proteína $X_{i}$, e os parâmetros da regressão são $b_{0}, b_{1}$ e $b_{2}$, e 0 termo referente a variação aleatória é o $e_{i j}$ estão linearmente dispostos.

A maioria dos modelos empregados em experimentação avícola são do tipo modelo linear, uma vez que eles são mais facilmente implementados nas análises estatísticas. Entretanto, muitos fenômenos biológicos são mais bem descritos por modelos não-lineares como os modelos que descrevem o crescimento das aves, como o aumento do peso em função do tempo, utilizando a função de Gompertz (Gous, 1986, 1998; Hruby et al., 1994; Emmans, 1995), cuja expressão é dada por:

$$
\left.W_{t}=W_{0} \exp \{L / K)\left(1-e^{-K t}\right)\right\}
$$

em que $W_{t}$ é o peso ao tempo $t, W_{0}$ é o peso no momento da eclosão, L é a taxa inicial de crescimento, e K é a taxa exponencial de redução do crescimento $L$.

Segundo Baldwin (1995), os modelos matemáticos ainda podem ser classificados como:

1) estáticos ou dinâmicos,

2) determinísticos ou estocásticos, e

3) como empíricos ou mecanísticos.

Os modelos estáticos descrevem o fenômeno em determinado momento ou instante, enquanto que nos modelos dinâmicos os parâmetros podem variar no tempo. Os modelos empregados nas determinações de exigências nutricionais para uma idade específica (NRC, 1994) podem ser classificados como estáticos, enquanto que as curvas de crescimento são exemplos de modelos dinâmicos.

Modelos determinísticos são aqueles que só têm uma única resposta, enquanto que nos modelos estocásticos (ou probabilísticos) possíveis respostas dependem de uma distribuição de probabilidade. Ao definir que as variáveis $\mathrm{e}_{\mathrm{ij}}$ e $\mathrm{e}_{\mathrm{ijk}}$ dos modelos (1), (2) e (3) podem assumir valores amostrados ao acaso de uma distribuição de probabilidade, esses modelos podem ser considerados como exemplos de modelos estocásticos. A distribuição normal (Figura 1) é a distribuição de probabilidade mais empregada nos modelos estocásticos das pesquisas avícolas.
0 modelo (4) é um exemplo de modelo determinístico, uma vez que para determinados parâmetros da curva de crescimento $\left(W_{0}, L\right.$ e $\left.K\right)$ e para determinada idade $(t)$ é obtida apenas uma resposta $\left(W_{t}\right)$, o que poderia representar o peso médio esperado de uma linhagem para a idade (t). Entretanto, adicionando um fator de variação aleatória $\left(\mathrm{e}_{\mathrm{i}}\right)$, obtém-se um modelo estocástico do tipo:

$$
\mathrm{W}_{\mathrm{ti}}=\mathrm{W}_{\mathrm{o}} \exp \left\{(\mathrm{L} / \mathrm{K})\left(1-\mathrm{e}^{-\mathrm{Kt}}\right)\right\}+\mathrm{e}_{\mathrm{i}}
$$

em que $W_{\text {ti }}$ pode ser interpretado como sendo o peso do animal i ao tempo t. Os modelos estocásticos permitem a estimação dos parâmetros de dispersão, os quais medem a variabilidade dos indivíduos que compõem a população.

$\mathrm{Na}$ área de nutrição e manejo, a maioria dos modelos de simulação disponíveis atualmente são determinísticos, enquanto que os modelos estocásticos são os mais usados nos estudos de melhoramento animal.

Os modelos empíricos são baseados apenas em correlações ou associações entre duas ou mais variáveis, sem levar em consideração os mecanismos que controlam o fenômeno. Por outro lado, os modelos mecanísticos tentam explicar ou descrever os mecanismos envolvidos, baseando-se nas leis da física, química, bioquímica etc.

A dificuldade em se definir os mecanismos envolvidos nos fenômenos biológicos faz com que a maioria dos modelos propostos para aves sejam empíricos. Os modelos geralmente só descrevem o crescimento ou a produção de ovos do ponto de vista da composição corporal ou do ovo (energia, proteína, gordura, cinzas ou aminoácidos). Entretanto, é muito importante tentar desenvolver modelos mecanísticos que descrevam fenômenos como o metabolismo de nutrientes, a digestão, o crescimento de tecidos, a postura e o desenvolvimento embrionário, para se aprofundar nos conhecimentos dos processos envolvidos (France \& Thornley, 1984; Baldwin, 1995; M cNamara et al., 2000).

O termo modelo também é adotado para a representação de um sistema, com diagramas de fluxo (Figura 2). Nesse caso, vários modelos matemáticos podem estar envolvidos na implementação dos diversos fatores que explicam a eficiência do sistema. Esses fatores são colocados em uma seqüência lógica de acontecimentos e a forma de implementação é variável em função do grau de conhecimento e interesse do pesquisador sobre determinados fatores e dos recursos computacionais. Esses tipos de modelos são chamados de modelos por compartimentos 
(Baldwin, 1995; Rosenblatt, 1998).

\section{Simulação em computador}

Na simulação em computador de fenômenos biológicos, os programas basicamente atribuem valores às variáveis quantitativas que representam o(s) fator(es), dispostos à direita do sinal de igual (=) do(s) modelo(s) matemático(s) eleito(s), produzindo resultados do fenômeno em estudo, representado à esquerda do sinal de igual.

A maneira em que os valores são atribuídos às variáveis dependem do grau de conhecimento do pesquisador e/ou programador. Considerando que o modelo (1) represente a influência do fornecimento de três tipos de ração $(i=1,2,3)$ sobre o peso corporal dos frangos, um programa de simulação pode atribuir valores prédeterminados para $m$, que representem a média de peso dos frangos, e para cada $t_{i}$, que represente a superioridade ou inferioridade de cada tratamento $\mathrm{i}$, enquanto que o valor atribuído para cada $\mathrm{e}_{\mathrm{ij}}$ pode ser obtido por meio de uma função que gera números aleatórios de acordo com a distribuição de probabilidade assumida.

Ao definir que uma variável pode assumir valores amostrais de uma distribuição de probabilidade, define-se o grau de incerteza a respeito da variável. No caso da distribuição normal, essa incerteza pode ser refletida pelo valor assumido para a variância, que tende a ser maior quanto maior for 0 desconhecimento sobre a variável.

Nos modelos determinísticos, nenhuma distribuição de probabilidade é assumida, podendose, teoricamente, predizer os resultados a partir do momento que os valores das variáveis forem definidas. Entretanto, em modelos complexos e/ou com grande número de níveis dentro dos fatores, a predição torna-se complexa e trabalhosa, aumentado o valor da simulação computacional.

Dependendo do modelo empregado, a descrição de um processo pode realizar-se em diferentes níveis (Baldwin, 1995; M cNamara, 2000). Um modelo pode ser empregado para descrever o crescimento total de um lote de frangos, de uma ave por vez, de um sistema fisiológico (massa muscular, tecido ósseo, sistema reprodutivo, trato digestivo), de um órgão (fígado, rim etc), de uma célula; ou o funcionamento de um processo fisiológico em cada um desses níveis.

Simulações de dados a partir de modelos simples, como os apresentados, podem ser realizadas em planilhas eletrônicas ou pacotes estatísticos disponíveis, com relativa facilidade. Entretanto, um programa de simulação pode ser a agregação de diferentes níveis de descrição de um fenômeno. Assim, a produção anual de uma granja pode ser estimada por um programa que integre vários modelos, os quais devem estar organizados, numa forma hierárquica, desde os processos bioquímicos celulares até a definição do número e peso dos lotes de abate.

Em cada nível de descrição pode existir uma abordagem mais apropriada. Por exemplo, alguns termos referentes à catálise das enzimas no nível celular podem ter pouca influência no peso da ave ao abate, enquanto que o peso corporal pode ser pouco informativo quando se estuda o crescimento da massa muscular em proteína.

Quanto mais detalhada for a descrição do processo, maior será o número de variáveis envolvidas no modelo, aumentando a complexidade do programa e a demanda computacional. Entretanto, a modelagem computacional pode ser o meio pelo qual o conhecimento obtido em fisiologia e metabolismo, através de minuciosos estudos do funcionamento de órgãos e células, seja utilizado para se evoluir o entendimento das funções animais e das fontes de variação do desempenho animal e finalmente no desenvolvimento de estratégias nutricionais, genéticas ou de manejo, que otimizem o retorno econômico (Baldwin, 1995).

A utilidade prática de um modelo de descrição de processos biológicos vai depender da sua interação dentro de um grande pacote que também contenha aspectos econômicos da indústria e da comercialização. Assim, modelos que descrevem processos econômicos, como os que descrevem a relação custo-receita, são importantes na modelagem de um sistema de produção. Por exemplo, um modelo para descrever os requerimentos nutricionais para poedeiras vai ser mais útil quando ele for integrado dentro de um programa de computador que:

1) Estabeleça a formulação a mínimo custo;

2) Otimize a estratégia de mercado e a produção mais rentável para a empresa;

3) Interprete as predições simuladas para identificar as razões de alguma ineficiência biológica e fazer recomendações estratégicas de manejo para melhorar a eficiência da empresa (M enzies et al., 1988);

4) Permita o armazenamento (Black, 1995) e a atualização (Fleming, 1987) dos dados;

5) A presente os resultados objetivos na forma de tabelas e gráficos entre outros. 


\section{Obtenção e avaliação dos modelos}

A credibilidade dos modelos empregados nas simulações depende desses estarem baseados em dados provenientes de pesquisas que empregam métodos científicos de experimentação, os quais requerem avaliações por meio de metodologias estatísticas apropriadas. Dentre os procedimentos estatísticos empregados nas experimentações, as análises de regressão linear, não-linear ou múltipla merecem um importante destaque por serem bastante úteis na determinação da relação existente entre as variáveis respostas e os fatores considerados nos modelos. A quantificação dessas relações é fundamental para o processo de implementação dos programas de simulação.

Os estudos de crescimento (modelos 1 e 2) e de exigências nutricionais (modelo 3 ) se beneficiam bastante das informações obtidas em análises de regressão (Curnow, 1986). Entretanto, é importante entender as vantagens e desvantagens de cada modelo de tal forma que seja possível obter o máximo benefício (Stilborn et al., 1994).

Para avaliação de um modelo, pode-se testá-lo em populações de comportamento conhecido ou com dados da literatura, além de outras formas. Segundo Harlow \& Ivey (1994), quatro aspetos básicos precisam ser avaliados em cada modelo: a calibração, a acurácia, a precisão e a viés.

A calibração é o processo de ajuste dos parâmetros que permite ao modelo estimar o crescimento em condições comerciais. Cada modelo utiliza um método de calibração diferente, podendo influenciar sua flexibilidade e/ou confiabilidade.

A acurácia de um modelo determina quão próximo o modelo estima os dados de produção. A precisão do modelo está relacionada à variabilidade de suas respostas. Um modelo pouco preciso pode apresentar resultados muito variáveis para uma mesma condição. 0 viés do modelo indica as falhas sistemáticas para predizer os valores, o que pode levar a super ou subestimação de variáveis importantes.

\section{Modelagem na determinação de exigências nutricionais}

A estimação das exigências nutricionais tradicionalmente fundamenta-se em experimentos que medem a resposta do animal, em ganho de peso ou conversão alimentar, como uma média de uma linhagem genética de aves, para um período específico, em condições ambientais e alimentares fixas. Isto indica que os valores de exigência de um nutriente são aplicáveis, com exatidão, para condições semelhantes ao que o experimento foi conduzido (Gous, 1998).

A implementação, em computador, de modelos de nutrição pode viabilizar a criação de sistemas interativos que disponibilizem informações de requerimentos para uma ampla variedade de condições.

Com a modelagem do crescimento, os nutricionistas podem predizer de maneira dinâmica as exigências de aminoácidos, proteína e energía de acordo com a taxa de crescimento ou de produção de ovos real das aves no momento da formulação (Hruby et al., 1994). Nesses programas, para se ter maior precissão nas formulações de rações, é necessário fornecer informações referentes às aves, ao alimento, ao meio ambiente, entre outros, de tal forma que se possa predizer o consumo de cada nutriente em determinada situação ambiental (Parks, 1982; Emmans \& Fisher, 1986; Emmans, 1981, 1995).

Assim, aumenta a cada dia a necessidade de se melhorar a descrição do desenvolvimento dos tecidos (músculos, penas e gordura), os quais são resultantes do potencial genético do animal e de suas interações com o consumo de nutrientes e demais fatores ambientais.

O estudo da curva de crescimento de cada animal, por meio de modelos estocásticos, tem se mostrado bastante útil, principalmente nas pesquisas de melhoramento animal. Com esses modelos, é possível ter uma melhor avaliação da população, podendo-se planejar maneiras de se promover mudanças desejáveis na forma da curva de crescimento dos animais, o que poderia ser feito por meio de manejos de nutrição e/ou seleção de animais. Assim, as estratégias de alimentação poderiam ser otimizadas (Gous, 1998).

Para interpretar os diferentes efeitos que os níveis de aminoácidos têm durante todo o ciclo de crescimento das aves, talvez seja necessário separar os efeitos de cada período de fornecimento desses nutrientes, de acordo com cada programa alimentar (dieta inicial, crescimento e final), nas curvas de crescimento (Zoons et al., 1991; Gous, 1998).

\section{Programas desenvolvidos}

Curnow (1973) desenvolveu um dos primeiros modelos de predição na Universidade de Reading. 0 Modelo de Reading determina os requerimentos em aminoácidos para maximizar os lucros, baseando-se na relação do custo dos aminoácidos com o valor do 
produto e o desvio padrão dos parâmetros resposta dos frangos.

Emmans $(1981,1987)$ propôs um modelo que utiliza curvas de crescimento para frangos de corte e particiona a resposta para energia e proteína dietética consumida em manutenção diária, crescimento de músculo e penas. O programa foi subseqüentemente comercializado para empresas de computação que produziram modelos como o M odelo FORTEL ${ }^{\text {TM }}$.

Na Universidade de Hebrew, foi desenvolvido por Hurwitz et al. $(1978,1980)$ e Talpaz et al. (1986) um modelo baseado nas determinações de exigências de aminoácidos e energia para crescimento e manutenção diária dos frangos. O custo dos alimentos foi estimado dos ganhos diários e utilizado para obter o menor custo no crescimento. 0 programa tem sido revisado e está comercialmente disponível como CHICKPOT $^{\mathrm{m}}$.

O modelo proposto por Pesti et al. (1986) foi baseado em equações quadráticas definidas em experimentos de crescimento de aves alimentadas com várias dietas. Foi empregado o conceito de que a ave estaria crescendo para um determinado peso alvo, dependendo do regime de alta ou baixa proteína ou energia. 0 produto final e a ingestão seriam determinados de acordo com a economia do sistema.

Desde 1989, a empresa NOVUS ${ }^{m}$ tem um programa de modelagem de crescimento para a indústria avícola. 0 modelo de crescimento de Ivey $\left(\right.$ IGM $\left.{ }^{\circledR}\right)$ tem sido amplamente aceito e está sendo utilizado extensivamente nos EUA, América Latina e Europa. 0 IGM ${ }^{\circledR}$ é baseado nas equações diferenciais para crescimento e quantifica os efeitos nutricionais e/ou ambientais que reduzem o desempenho para um ótimo teórico. 0 IGM ${ }^{\circledR}$ inclui um otimizador que permite a geração ilimitada de diferentes opções de "menor custo".

Paralelamente ao modelo IGM ${ }^{\circledR}$, Hruby et al. (1994) usaram a modelagem para predizer as exigências diárias de aminoácidos (g/M cal). Foram empregadas análises da proteína dos músculos e penas juntos e as estimativas de níveis de aminoácidos para manutenção, publicadas por Emmans \& Fisher (1986).

A combinação dos modelos de Hurwitz e o IGM permitiu o desenvolvimento do OmniPro $\|^{\circledR}$ (Novus, Inc.). Esse programa tem um alto nível de precisão e tem sido testado em larga escala com dados da indústria (Ivey, 1999). O modelo é calibrado com as curvas de crescimento das diferentes linhagens genéticas disponíveis comercialmente, curvas de crescimento observadas previamente na granja, sexo da ave, peso vivo e conversão alimentar, idade ao abate, temperatura ambiente, e programa alimentar (níveis nutricionais e idade de troca de alimentos). A calibração é fundamental para que o programa ajuste suas curvas de crescimento de acordo com as condições particulares de cada complexo de produção. Sem esse ajuste, bons níveis de precisão não poderiam ser obtidos nos passos seguintes de simulação e otimização.

A pós a calibração, o OmniPro ${ }^{\circledR}$ simula o crescimento diário dos animais e calcula as exigências nutricionais de energia, proteína e aminoácidos e consumo de ração. Baseado nisso, esse programa calcula os custos de produção e gráficos ajudam a visualizar tempos ótimos para o abate e os benefícios econômicos de cada dieta. Finalmente, o OmniPro também prediz a distribuição das partes da carcaça e formula as rações a fornecer de acordo com o tipo de otimização que seja escolhida: otimização por metro quadrado, por ave ou quilograma de peso vivo alojado, ou por quilograma por dia do ciclo.

Outro exemplo de modelo é aquele descrito por King (2001), que estima os efeitos das mudanças dos níveis de aminoácidos da dieta na composição da carcaça e no crescimento. Finalmente, a EFG Software (NatalÁfrica do Sul), Fisher \& Gous (2000) tem desenvolvido um sofware para determinar a ótima concentração de nutrientes num programa de alimentação de frangos de corte. O programa inclui um programa de formulação a mínimo custo, um modelo dinâmico de crescimento de frangos e um processo de otimização. As três partes do programa interatuam para gerar o programa alimentar que maximiza os beneficios econômicos de acordo com os objetivos de produção.

Apesar dos modelos de determinação de exigências nutricionais terem alcançado um alto grau de refinamento, atualmente são pouco empregados pela indústria avícola.

Os modelos também podem descrever os sistemas industrias de processamento de alimentos para as aves, os sistemas de abate, sistemas de manejo de excretas e de ambiente, e muitos outros aspectos.

Lokhorst (1996) utilizou o consumo diário de alimento, o consumo de água e a temperatura ambiente como variáveis de entrada para avaliar a produção diária de ovos, o peso do ovo, os ovos classificados como de segunda, ovos de cama, mortalidade acumulativa, peso corporal e uniformidade do lote de galinhas. Esse conjunto de variáveis permitiu determinar, com base nas mudanças diárias durante 0 ciclo de produção, quando um lote apresentava um problema produtivo 
significativo. As simulações e posterior avaliação deste modelo permitiram determinar a importância produtiva de reduzir a variação diária de temperatura no aviário.

As técnicas de modelagem também têm sido aplicadas para se avaliar estratégias de melhoramento genético (M uir, 1997). 0 trabalho de acompanhamento de populações por várias gerações, que poderia durar vários anos e ter altos custos, pode ser simulado em poucos minutos.

A modelagem nas espécies avícolas está apenas no início quando é comparada com os grandes avanços na modelagem de diferentes fenômenos e sistemas em bovinos e suínos (Korver \& Van Arendonk, 1988; Baldwin, 1995; Moughan \& Verstegen, 1995; McLeod, 2000; McNamara et al., 2000).

\section{CONCLUSÕES}

A modelagem computacional tem se mostrado como uma importante ferramenta na tomada de decisões para problemas de difícil resolução na avicultura, produzindo resultados eficientes em um curto prazo de tempo.
A modelagem permite a integração de conhecimentos das mais variadas áreas relacionadas ao problema em questão e, com isso, esses estudos podem ter uma grande abrangência. Conseqüentemente, para o estudos mais complexos é importante desenvolvimento de projetos que integrem pesquisadores tanto da área biológica, quanto das áreas humanas, exatas e tecnológicas.

A modelagem, impulsionada pelos avanços computacionais, pode se converter em uma ferramenta de uso cotidiano na indústria e na pesquisa avícola. Contudo, para que os resultados produzidos por simulação sejam confiáveis, é necessário o fornecimento de informações fidedignas, por meio de modelos adequados, os quais, em geral, são provenientes de experimentos com animais. Assim, observa-se que 0 sucesso da modelagem computacional depende dos resultados das experimentações tradicionais.

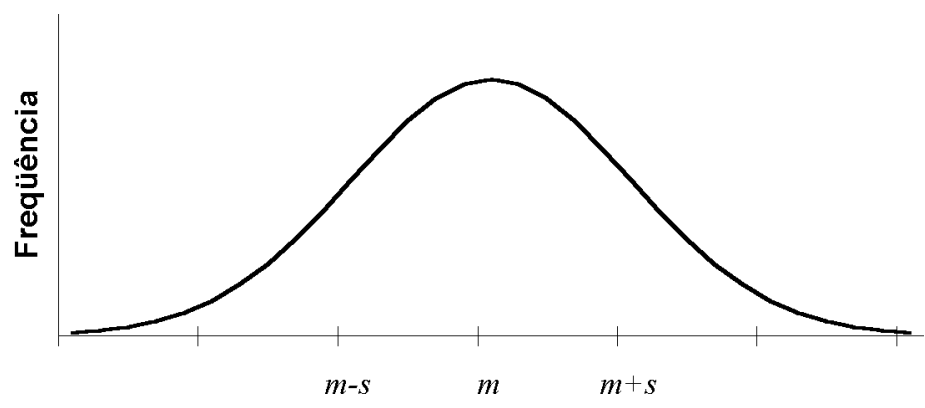

Figura 1- Representação gráfica de uma distribuição normal com média $\mathrm{m}$ e variância $\mathrm{s}^{2}$. 


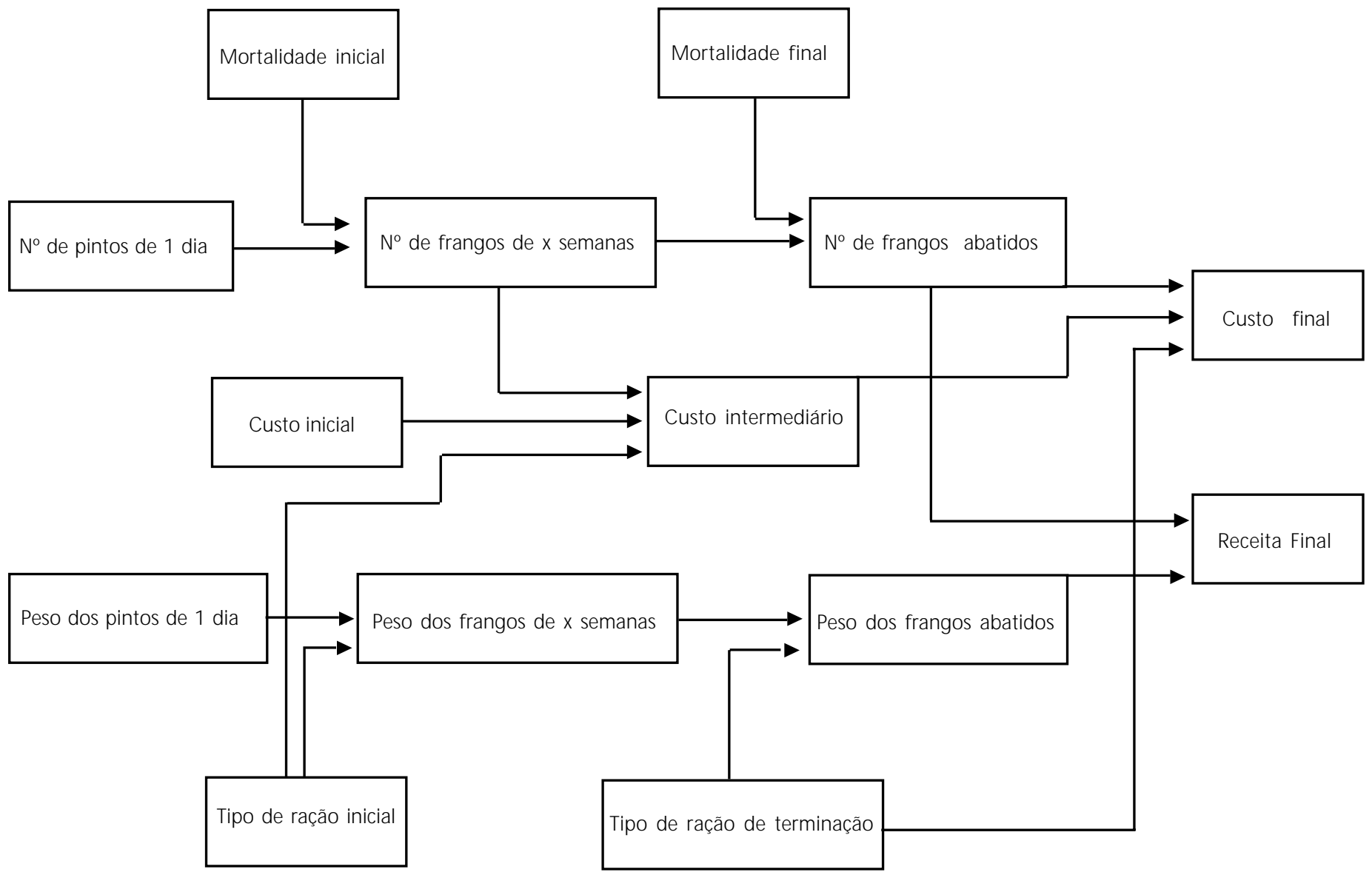

Figura 2 - Modelo de um sistema de produção de frangos de corte.

\section{REFERÊNCIAS BIBLIOGRÁFICAS}

Baldwin RL. Modeling ruminant digestion and metabolism. London (UK): Chapman \& Hall; 1995.

Black JL. The evolution of animal growth models In: Moughan PJ, Verstegen MWA, Visser-Reyneveld MI, editores. Modelling grow th in the pig. Wageningen (The Netherlands): Wageningen Pers; 1995 p. 3-9.

Curnow RN. A smooth population response curve based on an abrupt threshold and plateau model for individuals. Biometrics 1986; 29(1):1-10.

Emmans GC, Fisher C. Problems in nutritional theory. In: Fisher C, Boorman NK, editores. Nutrient Requirements of Poultry and Nutritional Research, British Poultry Science Symposium, London (UK): Butterworths; 1986. p. 9-39.

Emmans GC. A model of the growth and feed intake of ad libitum fed animals, particularly poultry. Pages 103-110. In: Computers in Animal production. Edinburgh, (Scotland): Occ.
Publ. No. 5, British Society of Animal Production; 1981.

Emmans GC. Growth, body composition and feed intake. World's Poultry Science Journal 1987; 43(3):208-227.

Emmans GC. Problems in modelling the growth of poultry. World's Poultry Science Journal 1995; 51(1):78-89.

Fisher C, Gous RM. Optimizing nutrient density in broiler feeds. In: World's Poultry Congress; 2000; Montreal, Quebec, Canadá. CD.

Fleming JF. Interface-Designer: Tools to assist the development of interactive software packages. In: White DH, Weber KM, editores. Computer assisted management of agricultural production systems. Werribee: Department of Agriculture and Rural Affairs; 1987. p. 152-154.

France J, Thornley JHM . Mathematical models in agriculture. London (England): Butterworth \& Co. Publishers; 1984. 
Gous RM. Making progress in the nutrition of broilers. Poultry Science 1998; 77(1):111-117.

Gous RM. M easurement of response in nutritional experiments. In: Fisher C, Boorman NK, editores. Nutrient Requirements of Poultry and Nutritional Research, British Poultry Science Symposium., London (UK): Butterw orths; 1986. p. 41-57.

Harlow HB, Ivey FJ. Accuracy, precision, and commercial benefits of growth modeling for broilers. Journal of Applied Poultry Research 1994; 3(4):391-402.

Hruby M, Hamre ML, Coon C. Growth modeling as a tool for predicting amino acid requirements of broilers. Journal of Applied Poultry Research 1994; 3(4):403-415.

Hurwitz S, Plavnik I, Bartov I, Bornstein S. The amino acid requirements of chicks: experimental validation of model calculated requirements. Poultry Science 1980; 59 (1):2470-2479.

Hurwitz S, Sklan D, Bartov I. New formal approaches to the determination of energy and amino acid requirements of chicks. Poultry Science 1978; 57(1):197-205.

Ivey FJ. Desenvolvimento e aplicação de modelos de crescimento para frango de corte. In: Simpósio internacional ACAV-Embrapa sobre Nutrição de Aves; 1999; Concórdia, Santa Catarina, Brasil. p. $22-35$.

King RD. Description of a growth simulation model for predicting the effect of diet on broiler composition and growth. Poultry Science 2001; 80(3):245-253.

Korver S, Van Arendonk JAM. M odeling of livestock production systems. Dordretcht (The Netherlands): Kluwer Academic Publishers; 1988.

Lokhorst C. Mathematical curves for the description of input and output variables of the daily production process in aviary housing systems for laying hens. Poultry Science 1996; 75(7):838-848.

M cLeod M G. M odelling the utilization of dietary energy and amino acids by poultry. In: Theodorou MK, France J, editores. Feeding systems and feed evaluation models. New York (NY):CABI Publishing; 2000. p. 393-412.

McNamara JP, France J, Beever D. M odeling nutrient utilization in farm animals. London (UK):CAB International; 2000.

Menzies TJ, Black JL, Dean M, Fleming JF. Combining heuristics and simulation models: an Expert System for the optimal management of pigs. In: "Al" 88 , Proceedings of the Australian Joint Artificial Intelligence Conference; 1988; Adelaide: University of Adelaide Printing Service; 1988. p. 176-189.

Moughan PJ, Verstegen MWA, Visser-Reyneveld MI. Modelling growth in the pig. Wageningen (The Netherlands): Wageningen Pers; 1995.

Muir MW. Genetic selection strategies: computer modeling. Poultry Science 1997; 76(8):1066-1070.
National Research Council. Nutrient Requirements of Poultry $9^{\text {th }}$ ed. Washington DC: National Academy Press; 1994.

Parks JR. A theory of feeding and growth of animals. New York (NY):Spinger-Verlag; 1982.

Pesti GM , Arraes RA, M iller BR. Use of the quadratic growth response to dietary protein and energy concentrations in least cost feed formulation. Poultry Science 1986; 65(6):1040-1051.

RosenblattJ. The mathematics behind modeling. In: Clifford AJ, M üller Hans-Georg, editores. Mathematical modeing in experimental nutrition. New York (NY):Plenum Press; 1998. p. 115-129.

Spedding CRW. General aspects of modelling and its application in livestock production In: Korver S, Van Arendonk JAM, editores. Modelling of livestock production systems. Brussels (Belgium): Kluwer Academic Publishers; 1988. p. 3-9.

Stilborn HL, M oran Jr ET, Gous RM, Harrison MD. Experimental data for evaluating broiler models. Journal of Applied Poultry Research 1994; 3(4):379-389.

Talpaz H, Torre JR de la, Sharpe PJH, Hurwitz S. Dynamic optimization model for feeding of broilers. Agricultural Systems 1986; 20(1):121-132.

Zoons J, Buyse J, Decuypere E. Mathematical models in broiler raising. World's Poultry Science Journal 1991; 47(3):243-255. 